\section{Expert Opinion}

1. Introduction

2. CD133 as a stem cell marker

3. Blood derived $\mathrm{CD} 133^{+}$cells

4. Muscle derived $\mathrm{CD} 133^{+}$cells

5. Therapeutic applications of $\mathrm{CD} 133^{+}$cells

6. Heterogeneity of $\mathrm{CD}_{133^{+}}$stem cells

7. Expert opinion

\section{informa}

\section{healthcare}

\section{CD133 ${ }^{+}$cells isolated from various sources and their role in future clinical perspectives}

\author{
Mirella Meregalli, Andrea Farini, Marzia Belicchi \& Yvan Torrente ${ }^{\dagger}$ \\ Stem Cell Laboratory, Department of Neurological Sciences, Università degli Studi di Milano, \\ Fondazione IRCCS Cà Granda Ospedale Maggiore Policlinico, Centro Dino Ferrari, via Francesco \\ Sforza 35, Milan, Italy
}

Background. CD133 is a member of a novel family of cell surface glycoproteins. Initially, the expression of CD133 antigen was seen only in the hematopoietic derived $\mathrm{CD}_{3} 4^{+}$stem cells. At present, CD133 expression is demonstrated in undifferentiated epithelium, different types of tumors and myogenic cells. $\mathrm{CD}_{133^{+}}$neurosphere cells isolated from brain are able to differentiate into both neurons and glial cells. These data suggested that CD133 could be a specific marker for various stem and progenitor cell populations.

Objectives. The main goal would be to describe the role for CD133 as a marker of stem cells able to engraft and differentiate, to form functional nonhematopoietic adult lineages and contribute to disease amelioration via tissue regeneration.

Results/conclusion. In conclusion, since the rise of CD133 antigen as a suitable stem cell marker, the possible use of $\mathrm{CD}_{133^{+}}$stem cells in therapeutic applications has opened a new promising field in the treatment of degenerating diseases. The human circulating cells expressing the CD133 antigen behave as a stem cell population capable of commitment to hematopoietic, endothelial and myogenic lineages. CD133 cell therapy may represent a promising treatment for many diseases.

Keywords: CD133+, DMD, prominin-1, stem cells

Expert Opin. Biol. Ther. (2010) 10(11):1521-1528

\section{Introduction}

In the last years, stem cells have received much attention for their potential use in cell-based therapies for various human diseases; according to these observations, various stem cells were used to treat muscular dystrophy. CD133 is a member of a novel family of cell surface glycoproteins [1-3] and it was recognized to be the homologue of mouse prominin- 1 . Human prominin-1 is a transmembrane glycoprotein of 865 amino acids with a total molecular mass of $120 \mathrm{kDa}$; it has a unique structure consisting of an $\mathrm{N}$-terminal extracellular domain, five transmembrane domains with two large extracellular loops and a 59 aa cytoplasmic tail. Transcription of CD133 is driven by five alternative promoters, three of which are located in a $\mathrm{CpG}$ island and at least partially regulated by methylation [4]. The transcripts contain one of five alternative 5'-UTR first exons, which are expressed in a tissue-specific manner [4]. The function of CD133 is not known, and its ligand has not yet been identified. At first, the expression of CD133 antigen was seen only in the hematopoietic system-derived $\mathrm{CD}_{3} 4^{+}$stem cells, isolated from fetal liver, bone marrow and peripheral blood $[2,3]$. These cells were successfully injected in animal models suggesting that they had a long-term repopulating potential [3]. Later, CD133 expression was demonstrated on undifferentiated epithelium [5], retinoblastoma [2], teratocarcinoma [2], leukemias [6,7], myogenic cells [8] and endothelial progenitors 
expressing VEGFR-2 and fetal brain neural stem cells [9]. In particular, CD133 was best characterized for identifying normal brain stem cells [9] and tumor propagating cells (TPCs) in brain tumors [10] and also used as a marker for colon cancer TPCs [11]. In this context, an unusual relationship between promoter DNA methylation and CD133 expression was noted: further studies demonstrated a role for promoter methylation in the regulation of CD133 levels. Moreover CD133 neurosphere cells differentiated into both neurons and glial cells, and single $\mathrm{CD}_{13} 3^{+}$neurosphere cells were able to re-establish neurosphere cultures, demonstrating the selfrenewal potential of this highly enriched population [12]. Increasingly, studies used CD133 as a marker to identify cancer stem cells from a variety of tumors (Figure 1E, 1F). However, Shmelkov's recent work has raised serious concerns over the use of CD133 as a general marker for cancer stem cells. In this study [13], both $\mathrm{CD} 133^{+}$and $\mathrm{CD} 133^{-}$metastatic colon cancer cells $\mathrm{EpCAM}^{+}$showed long-term tumorigenesis in a NOD/SCID mouse model. This is different from what was found in two previous studies in colon cancer [11,14]. Actually, there are no convincing explanations for this significant controversy. Shmelkov et al. argued that the CD133- cells used in the previous studies consisted of stromal cells, endothelial cells but not tumorigenic cells of epithelial origin. Another important issue is the variation in tissue sampling. Even in the same type of cancer such as colon cancer, cells are affected by the location where samples are taken (e.g., center or periphery of a tumor), histopathological types, tumor stages and treatments (drugs, irradiations). In fact, cancer stem cell behavior is influenced by the surrounding microenvironment (niche), which is affected by tumor sizes, nutritional conditions, oxygen levels, chemotherapies and irradiations [14-16]. Based on these controversial evidences, it seems to be inappropriate to use CD133 as a general cancer stem cell marker, particularly in tissues where CD133 is expressed in epithelial cells and epithelial stem cells, and their counterpart cancer cells and cancer stem cells.

These data suggested that CD133 could be a specific marker for various stem and progenitor cell populations. This review focuses on the possibility of using $\mathrm{CD} 133^{+}$stem cells isolated from blood and muscle in cell-mediated therapies in order to specify the clinical potentiality of this kind of stem cells and their role in future clinical perspectives. Moreover, the review describes a good number of clinical trials demonstrating the applicability of these cells in different fields rather than muscle pathologies.

\section{CD133 as a stem cell marker}

CD133 antigen is expressed by hematopoietic progenitors [3]; this evidence has directed interest towards the potential of CD133 as a cell surface marker of adult stem cells. In hematopoietic lineages in humans, CD133 antigen expression is restricted to $\mathrm{CD} 34^{+}$cells, although $\mathrm{CD} 133$ transcripts have been found in many human cell lines and differentiated cells [18].
A role for CD133 as a marker of stem cells with the capacity to engraft and differentiate to form functional non-hematopoietic adult lineages and contribute to disease amelioration via tissue regeneration is emerging, and $\mathrm{CD} 133^{+}$cell therapy may represent a promising treatment for many diseases. Human $\mathrm{CD}_{133^{+}}$cells, isolated from human peripheral blood and manipulated in vitro to undergo myogenesis, were shown to ameliorate disease via a direct contribution to muscular regeneration when transplanted into scid/m $d x$ dystrophic mice, a model of Duchenne muscular dystrophy (DMD) [8]. DMD, the most common form of muscular dystrophy, is an X-linked genetic disorder due to either spontaneous mutations or inherited nonsense point mutations in the dystrophin gene [19]. Dystrophin is responsible for the maintenance of cell integrity and muscle cell function [20]. The loss of a functional dystrophin protein gives rise to progressive muscle degeneration in DMD patients as a result of mechanical stress during contraction that leads to death in the second second/third decade of the patient's life [21]. Human circulating $\mathrm{CD} 133^{+}$cells were also induced to undergo either endothelial or cardiomyocytic differentiation in vitro [8]. In addition to the hematopoietic system, CD133 is also expressed on endothelial progenitor cells (EPCs), which play a role in angiogenesis and neovasculogenesis during both tumor growth and wound healing [23]. In a clinical point of view, $\mathrm{CD} 133^{+}$stem cells are to migrate into the vasculature. In 2006, we discovered the mechanisms involved in the muscle homing of $\mathrm{CD}_{133^{+}}$stem cells, allowing the systemic delivery of such stem cells. Almost all $\mathrm{CD} 133^{+}$cells co-expressed CD44 and LFA-1, while $>40 \%$ of the CD133 cells expressed PSGL-1, VLA-4, L-selectin and CCR7. The expression of this pattern of adhesion molecules gave to $\mathrm{CD} 133^{+}$stem cells the potentiality to mediate the migration through the blood vessel wall. We also showed the expression of murine endothelial adhesion molecules potentially involved in dystrophic muscle homing, such as ICAM-1, VCAM-1, P-selectin and E-selectin [23]. As all these studies were carried out in the frame of a more general research program aiming to use human stem cells to repair muscle damage in muscular dystrophies, such as DMD, it was clear that the elucidation of the mechanisms involved in muscle homing of stem cells could aid in improving a potential therapy for those diseases. In this sense, combining nanoparticle labeling of blood-derived $\mathrm{CD} 133^{+}$cells and the X-ray computed microtomography, we provided detailed information on the stem cell migration in 3D. This is of special importance if the cells are administered systemically rather than directly into the target tissue. Intra-arterially administered labeled CD133 stem cells were distributed around the vessels of muscle tissues. It is very important in the future to combine cell therapy and innovative technology in order to study the distribution of the injected cells.

\section{Blood derived CD133+ cells}

In the paper published in 2004, we obtained the first evidence that $\mathrm{CD} 133^{+}$cells isolated from peripheral blood exhibited 

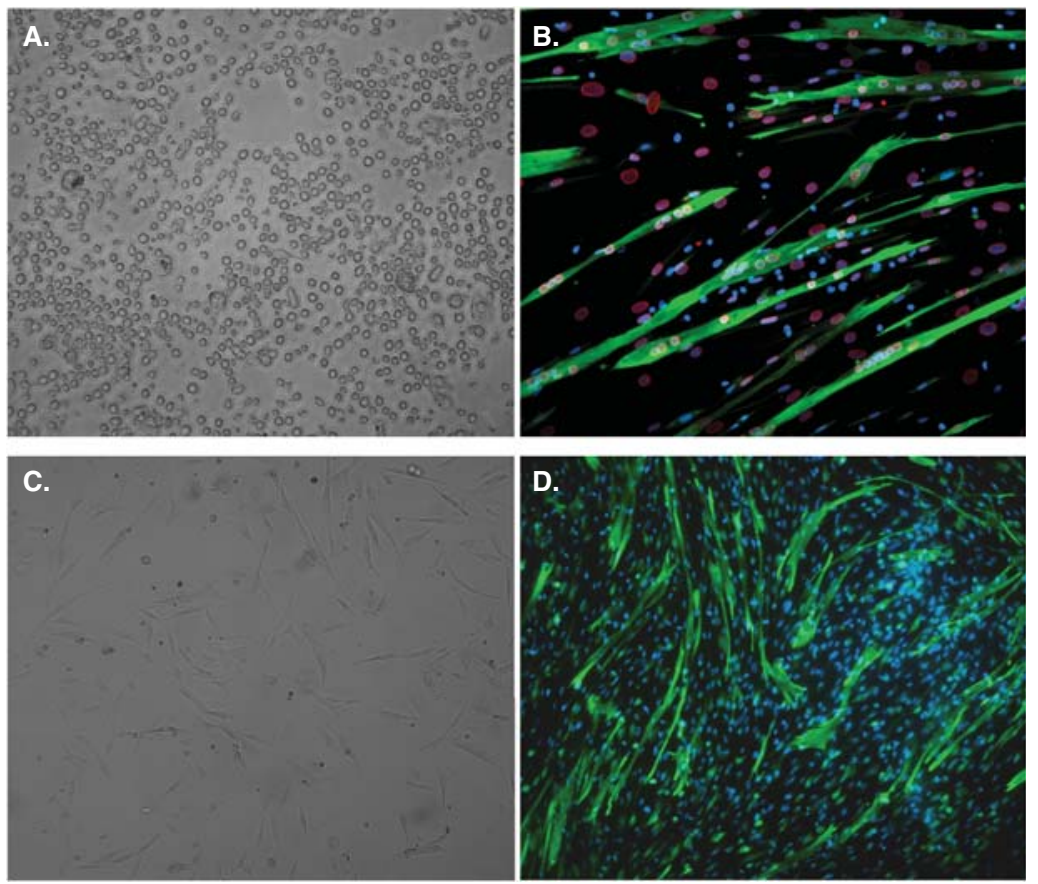

Figure 1. $\mathrm{CD}_{133^{+}}$cells isolated from peripheral blood in proliferation medium A.; blood derived $\mathrm{CD} 133^{+}$cultured in the presence of a feeder layer of murine myotubes fused (human nuclei stained with lamin $A / C$ antibody in red) and formed MyHC-expressing heterozygous-specific human/murine myotubes (green) B.; muscle-derived CD133 ${ }^{+}$cells in culture C.; in the proliferation medium, muscle derived $\mathrm{CD}_{133^{+}}$cells were to differentiate into multinucleated myotubes expressing MyHCs (green) D.

MyHC: Myosin heavy chain.

stem cell behavior (Figure 1A) [8]. The cloned CD133-derived stem cells were able to commit to more than one lineage, such as hematopoietic and endothelial. In particular, we demonstrated that circulating $\mathrm{CD} 133^{+}$cells were able to differentiate into myotubes in vitro and to participate in skeletal muscle regeneration in a murine model of DMD, the scid/mdx mouse, expressing several myogenic markers such as MYF5, $\mathrm{m}$-cadherin and Pax7. Moreover, when these cells were cultured in the presence of a feeder layer of mouse myogenic cells, they fused and formed myosin heavy chain (MyHC)expressing heterozygous-specific human/murine myotubes (Figure 1B) [8]. Following the in vitro characterization, we assessed the in vivo behavior of $\mathrm{CD} 133^{+}$stem cells isolated from blood in term of capacity to restore dystrophin expression and eventually regenerate the satellite cells pool in scid/ $m d x$ mouse after intramuscular and intra-arterial delivery. Human $\mathrm{CD}_{133^{+}}$cells colonized the mouse muscle and formed hybrid regenerated fibers expressing human dystrophin. When transplanted into dystrophic skeletal muscle, human cells differentiated into satellite cells; in fact, they were located beneath the basal lamina and distributed along freshly isolated fibers, co-expressing $\mathrm{m}$-cadherin. After intraarterial injections, $\mathrm{CD}_{133^{+}}$donor cells were detected in several vessels near areas of regeneration, where they expressed human ve-cadherin and CD31 [8]. According to these evidences, we suggested that human blood-derived CD133 ${ }^{+}$ cells migrated within muscle tissues and differentiated into endothelial, satellite cells and human dystrophin-positive muscle fibers. Moreover, we noted a significative recovery of force after treatment with a value similar to the normal mice [8].

Other researchers demonstrated that autologous $\mathrm{CD} 133^{+}$ bone marrow-derived cells induced angiogenesis and restored myocardial tissue viability after infarction, as discussed in detail below $[25,26]$.

\section{Muscle derived $C D 133^{+}$cells}

As we isolated myogenic CD $133^{+}$from blood, we assessed to identify a similar population from muscle (Figure 1C). We identified a subpopulation of stem cells expressing the CD133 together with CD34, CD45 and KDR from human normal and DMD biopsies. Muscle-derived $\mathrm{CD}_{133^{+}}$cells were present in both normal and DMD muscles, although dystrophic muscle biopsies of young patients $(5-14$ years old) contained a higher number of these cells than muscle biopsies of healthy donors matched for age [27]. They represented $2 \%$ of the total dystrophic muscle-derived nucleated cells. Both normal and dystrophic muscle-derived CD133 ${ }^{+}$ cells expressed the CD45 antigen (4\%), indicating their 
hematopoietic commitment [27]. Furthermore, the expression of Pax-7, Myf-5, MyoD, m-cadherin, MRF-4 and myogenin after 24 days of culture in the proliferation medium and their ability to differentiate into multinucleated myotubes expressing $\mathrm{MyHCs}$ suggested a myogenic commitment (Figure 1D). As we assessed the capacity of proliferation and differentiation of $\mathrm{CD}_{133^{+}}$stem cells isolated from normal and dystrophic muscle biopsies, we decided to consider the musclederived $\mathrm{CD} 133^{+}$cells as a class of myogenic precursors. In a clinical point of view, we investigated their safety after transplantation in DMD muscles [26].

Our data showed that intramuscular transplantation of muscle-derived $\mathrm{CD} 133^{+}$cells in DMD patients was a safe procedure and feasible [26]. Following the injection of $\mathrm{CD} 133^{+}$stem cells, the patients showed an increased number of capillaries per muscle fiber and expressed a change in the ratio of slow:fast myosin myofibers.

\section{Therapeutic applications of $\mathrm{CD} 133^{+}$cells}

Since the rise of CD133 antigen as a suitable stem cell marker, the possible use of $\mathrm{CD} 133^{+}$stem cells in therapeutic applications opened a new promising field in the treatment of degenerating diseases. Bhatia et al. demonstrated that transplanted $\mathrm{CD} 133^{+} \mathrm{CD} 34^{-}$cells resulted in similar repopulating potentials as $\mathrm{CD}_{3} 4^{+}$cells and can differentiate into $\mathrm{CD} 133^{+}$ CD $34^{+}$cells with hematopoietic and endothelial capacity [27]. Following hematopoietic stem cell transplantation, early clinical studies showed slightly improved engraftments with $\mathrm{CD} 133^{+}$cells compared to $\mathrm{CD} 34^{+}$cells [29]. In addition, as described above, our results demonstrated that circulating $\mathrm{CD}_{133^{+}}$cells differentiated in myogenic cells and might be used for stem cell therapy of muscular dystrophy [8]. Stamm et al. showed that transplanted bone marrowderived $\mathrm{CD} 133^{+}$cells improved function of infracted myocardium probably as a result of the amelioration in blood vessel formation [29]. Despite these considerations, the isolation of $\mathrm{CD} 133^{+}$stem cells provided a selective population useful for transplantation and tissue regeneration as well as targeting population of malignantly transformed stem cells in certain cancers $[8,26]$. We extended these observations to a combination of cell- and gene-based approaches via the ex vivo introduction of corrective genes into dystrophic $\mathrm{CD} 133^{+}$ myogenic cells which would permit their subsequent autologous transplantation. Use of the patient's own cells would reduce the risk of implant rejection. As a correction method, we used the exon skipping approach. This technology uses specific antisense oligonucleotides designed to mask the putative splicing sites of exons in the mutated region of the primary RNA transcript whose removal would re-establish a correct reading frame. The $\Delta 49-50$ DMD deletion was chosen to test the capacity of the exon-skipping strategy to restore open reading frame to the dystrophin mRNA sequence in $\mathrm{CD} 133^{+}$stem cells [30]. Theoretically, skipping of exon 51 in this context should eliminate the original frameshift, revealing a correct reading frame between exons 48 and 52 . Both stem cell populations were transduced with a lentiviral vector expressing the U7 snRNA containing antisense sequences designed specifically to skip exon 51 and able to restore open reading frame to a range of DMD genotypes. In accordance with our hypothesis, the genetically engineered DMD stem cells expressed a shortened dystrophin mRNA specifically deleted for the exon 51 [30]. Our results also showed that human genetically engineered DMD blood and musclederived $\mathrm{CD} 133^{+}$can become located beneath the basal lamina and distributed along freshly isolated fibers where they express M-cadherin: all are indications that they can differentiate into satellite cells within the recipient dystrophic skeletal muscle [30]. However, intramuscular transplantations lead only to local and focused regeneration whereas DMD pathology affects the whole body musculature and its effective treatment requires methods to distribute the injected cells to these dispersed sites. This problem might be circumvented by intraarterial injection as a means of obtaining more dispersed delivery of $\mathrm{CD} 133^{+}$stem cells into dystrophic individuals.

Another important question that emerged with the $\mathrm{CD} 133^{+}$cells injection - unfortunately, common to a lot of different types of stem cells - is the self-renewal potential of the $\mathrm{CD} 133^{+}$transplanted cells. Although it is known that these cells are able to differentiate in vitro and participate to ameliorate the dystrophic phenotype of animal model after their transplantation $[8,26,30]$, it is not clearly known how long $\mathrm{CD} 133^{+}$isolated from muscle and blood can contribute to host tissue regeneration. However, it was previously published that $\mathrm{CD} 133^{+}$cells were able to exhibit myogenic properties 60 days [30] after transplantation in scid/mdx mice. Moreover, it could be very important to determine how many times cellular transplantation is performed to have better results in terms of efficiency of muscular development. Various studies are now in progress trying to find answers to these important questions, above all for a possible future therapeutic application of $\mathrm{CD} 133^{+}$stem cells.

Ongoing Phase I/II studies try to assess the efficacy and the safety of the injection of $\mathrm{CD} 133^{+}$stem cells isolated from different tissues applying to human diseases such as ischemia, refractory coronary heart disease, cirrhosis and myocardial infarction. In case of ischemia, the primary objective is to analyze the safety and efficacy of $\mathrm{CD} 133^{+}$cells obtained from peripheral blood in the treatment of diabetic patients with critic ischemia in lower limbs. The secondary objectives are: to determine the safety of the intramuscular administration of $\mathrm{CD} 133^{+}$cells that have been mobilized from peripheral blood, to determine the $\mathrm{CD} 133^{+}$capacity to increase the revascularization at lower limbs in diabetic patients with critic ischemia in the lower limbs and to evaluate the patient's global health. The majority of studies tries to assess the efficacy of an autologous transplantation of $\mathrm{CD}_{13} 3^{+}$in heart pathologies; one of these studies evaluates if the intracoronary infusion of autologous bone-marrow derived $\mathrm{CD}_{133^{+}}$endothelial precursor cells is able to promote 
neo-vascularization and improve myocardial perfusion and contractility in patients with refractory coronary heart disease, characterized by a poor response to standard coronary interventions, severe impairment of the quality of life and a poor prognosis. A completed study demonstrated the safety of intracoronary injection of $\mathrm{CD}_{133^{+}}$cells from autologous bone marrow (ABM) and mobilized peripheral blood $(\mathrm{MPB})$ in the target population and the efficacy, selective injection of $\mathrm{CD} 133^{+}$cells from $\mathrm{ABM}$ and $\mathrm{MPB}$ in the culprit vessel of the target population, and regional and global contractile function and perfusion and metabolism of the infarcted area. Limsuwan et al. published that a young patient suffering from myocardial infarction was transplanted with bone marrow-derived $\mathrm{CD}_{133^{+}} \mathrm{CD} 4^{+}$cells, showing improvement in functional capacity [25]. Although the amelioration of myocardium could be in part due to the daily injection of G-CSF, this condition is probably the consequence of the ability of injected cells to contribute to cardiac repair in terms of induction of angiogenesis, inhibition of apoptosis and promotion of myocite recovery.

In another work, Flores-Ramirez et al. demonstrated that a single coronary infusion of $\mathrm{CD}_{133^{+}}$EPCs into the infracted area improved heart function for 2 years after the treatment and remarkably improved the quality of life in treated patients [31]. Moreover, the intracoronary infusion of these cells seemed to be a minimally invasive, safe and less expansive method of treating patients with limited therapeutic options. Unfortunately, this study was limited by the small numbers of patients and the lack of control subjects. In addition, Navarro-Sobrino et al. showed that patients suffering from stroke mobilized an EPC fraction $\left(\mathrm{CD} 133^{+} \mathrm{CD}^{+} 4^{+} \mathrm{KDR}^{+}\right.$ $\left.\mathrm{CD} 45^{+}\right)$characterized by a great capacity to induce vasculogenesis and secrete angiogenic factors [32]. Now, fifteen ongoing Phase I and II studies are started using CD $133^{+}$stem cells (www.clinicaltrials.gov).

Although blood and muscle-derived $\mathrm{CD}_{133^{+}}$cells are largely used in different fields to regenerate pathological tissues, several things need to be ameliorated to consider these cells suitable for cellular therapy. We need to enhance the rate of proliferation of blood-derived $\mathrm{CD} 133^{+}$cells in culture and their storage for repeated treatments. We have to understand why blood-derived CD $133^{+}$cells are less efficient compared with muscle-derived $\mathrm{CD}_{133^{+}}$cells in terms of contribution of muscle nuclei. At the end, we have to ameliorate the strategy to deliver myogenic cells chronically to the various sites of sporadic regeneration that occur in muscular dystrophies.

\section{Heterogeneity of $\mathrm{CD} 133^{+}$stem cells}

Extraordinary progress in the understanding of several key features of stem cells has been made in the last 10 years, including definition of the niche, identification of signals regulating mobilization and homing as well as partial understanding of the mechanisms controlling self-renewal commitment and differentiation [33]. This progress has produced invaluable tools for the development of rational cell therapy protocols that have yielded positive results in preclinical models of genetic [34] and acquired diseases and, in several cases, have entered clinical experimentation with a positive outcome [35].

The research is an important step towards understanding stem cell behavior, how cellular development is controlled, and how a single cell - which has the genetic code within it to become any cell in the body - is told what to become, where to go and what metabolic function to perform.

A major limitation in these studies is the heterogeneity of stem cell populations obtainable by commercialized stem cells isolation methods [36]. The recruitment to myogenesis of resident and circulating progenitors can be strongly enhanced, being central in the support of muscular tissue functionality. $\mathrm{CD}_{133^{+}}$cells have been characterized for their regenerative potential in vivo as well as their ability to repopulate the satellite cell niche and they could be stratified for the expression of CD34 antigen identifying a subpopulation displaying high myogenic potential in vitro and in vivo. Probably, CD133 ${ }^{+}$ cells represent a heterogeneous population which includes a common progenitor with round shape, low proliferative capacity and potential to give rise to myogenic, endothelial and pericyte cells. Negroni et al. demonstrated that more human mononuclear cells remained in the interstitial space in the muscle injected with $\mathrm{CD} 133^{+} / \mathrm{CD} 34^{+}$cells when compared with the human myoblasts [37]. The percentage of undifferentiated interstitial cells confirms that all $\mathrm{CD}_{133^{+}}$ stem cells are not at the same stage of myogenic commitment and that some of the cells never go to terminal differentiation [37]. These evidences confirm that $\mathrm{CD} 133^{+}$cells are a heterogeneous population and contain a fraction already committed to myogenic differentiation giving rise to myonuclei and satellite cells and a fraction with slower capacity to differentiate improving regeneration by secreting factors that stimulate the amelioration of the muscle regeneration when injected in a dystrophic behavior [37].

$\mathrm{CD}_{133^{+}}$cells and mesoangioblasts can display similar behaviors in experimental conditions: mesoangioblasts [38] and $\mathrm{CD}_{133^{+}}$cells [23] have the ability to migrate through the vasculature rendering these cells good candidates for cell therapy. These evidences suggest that there is a probable connection between the origin and the development of these two kinds of progenitor cells. Such an event represents a clear explanation of the quantitative incongruence highlighted by the work of Grounds and McGeachie, demonstrating that the number of resident satellite cells is much smaller that the number of committed myogenic precursors that populate the muscle tissue soon after injury [39]. Moreover, it is unclear why all these progenitor cells are not able to defeat or at least ameliorate the symptoms of the disease. We propose that these committed progenitors were the steps of differentiation of a common un-differentiated stem cell whose function is largely unknown. 


\section{Expert opinion}

Stem cell therapy is an attractive method to treat human pathologies because only a small number of cells, together with a stimulatory signal for expansion, are required to obtain a therapeutic effect [40]. Different ongoing and concluded clinical trials demonstrated the applicability of $\mathrm{CD} 133^{+}$ stem cells particularly in the treatment of ischemia, refractory coronary heart disease, cirrhosis and myocardial infarction. Recently, promising results were published showing improvement in functional capacity in case of treatment of patients affected by heart pathologies [25,31]. In particular, we studied the clinical potential of these cells isolated from blood and muscle in the treatment of muscular dystrophy $[8,30]$. We know that the clinical relevance candidate stem cell population must be easily extracted, must remain capable of efficient myogenic conversion and when transplanted must integrate into the muscles allowing the functional correction of the dystrophic phenotype [40]. Survival and the migration from the site of injection to the compromised muscles of the body remain suboptimal for many of the cell populations. We demonstrated that human circulating cells expressing the CD133 antigen behave as a stem cell population capable of commitment to hematopoietic, endothelial and myogenic lineages and the discovery of the mechanisms involved in the muscle homing of stem cells improved a potential therapy for muscular dystrophy based on the systemic delivery of this population of stem cells [23].

$\mathrm{CD} 133^{+}$cells seem to satisfy the most criteria for use in the treatment of muscular dystrophy. Nevertheless, several issues have to be clarified such as the time required in culture for autologous cells before re-implantation into the patients and the contribution to muscle nuclei of blood- and musclederived $\mathrm{CD} 133^{+}$cells and the time that these cells engraft the satellite cell compartment, allowing the formation of genetically corrected stem cells into the muscle. Although satellite cells remain the primary cells responsible for the regeneration of post-natal skeletal muscle, several other cell types, such as MDSCs, $\mathrm{CD} 133^{+}$cells and mesoangioblasts, can display similar behavior in experimental conditions. As we explained before, it is very important to clarify the possibly common origin of these stem cell populations, their relationship to satellite cells and whether these myogenic cells have the potential to give rise to muscular cell lineages also in steady-state conditions [41]. It is expected that a robust and thoroughly tested model of gene networks involved in stemness will be instrumental in the identification of a novel stem cell isolation method able to produce a 'pure' stem cell population. We strongly believe that combining mathematical modeling with computational and experimental approaches will efficiently tackle this problem, thus, bringing a holistic understanding of the molecular mechanisms that maintain stem cells in an undifferentiated state or on the contrary result in their engagement into a specific differentiation pathway. Understanding what defines stemcellness may have a profound impact also in understanding the role of circulating rare stem cells in normal or pathological conditions. For the large economical burden that they represent, it is critical to develop innovative approaches and to explore novel avenues of research that will be instrumental in discovering new biomarkers and cures for cancer pathologies and other diseases such as hematopoietic and muscle wasting.

\section{Acknowledgments}

This paper has been sponsored by the Association Monégasque contre les Myopathies (AMM), Association Française contre les Myopathies (AFM), Associazione La Nostra Famiglia Fondo DMD Gli Amici di Emanuele, Associazione Amici del Centro Dino Ferrari, Fondazione IRCCS Ospedale Maggiore Policlinico and the European Union Framework Programme 223098.

\section{Declaration of interest}

The authors declare no conflict of interest and have received no payment in preparation of this manuscript. 


\section{Bibliography}

1. Corbeil D, Roper K, Fargeas CA, et al. Prominin: a story of cholesterol, plasma membrane protrusions and human pathology. Traffic 2001;2:82-91

2. Miraglia S, Godfrey W, Yin AH, et al. A novel five-transmembrane hematopoietic stem cell antigen: isolation, characterization, and molecular cloning. Blood 1997;90:5013-21

3. Yin AH, Miraglia S, Zanjani ED, et al. AC133, a novel marker for human hematopoietic stem and progenitor cells. Blood 1997;90:5002-12

4. Shmelkov SV, Jun L, St Clair R, et al. Alternative promoters regulate transcription of the gene that encodes stem cell surface protein AC133. Blood 2004;103:2055-61

5. Corbeil D, Roper K, Hellwig A, et al. The human AC133 hematopoietic stem cell antigen is also expressed in epithelial cells and targeted to plasma membrane protrusions. J Biol Chem 2000;275:5512-20

6. Buhring HJ, Seiffert M, Marxer A, et al. AC133 antigen expression is not restricted to acute myeloid leukemia blasts but is also found on acute lymphoid leukemia blasts and on a subset of CD34+ B-cell precursors. Blood 1999;94:832-3

7. Kratz-Albers K, Zuhlsdorp M, Leo R, et al. Expression of a AC133, a novel stem cell marker, on human leukemic blasts lacking CD34-antigen and on a human CD34+ leukemic line:MUTZ-2. Blood 1998;92:4485-7

8. Torrente Y, Belicchi M, Sampaolesi M, et al. Human circulating AC133(+) stem cells restore dystrophin expression and ameliorate function in dystrophic skeletal muscle. J Clin Invest 2004:114:182-95

9. Uchida N, Buck DW, He D, et al. Direct isolation of human central nervous system stem cells. Proc Natl Acad Sci USA 2000;97:14720-5

10. Singh SK, Hawkins C, Clarke ID, et al Identification of human brain tumour initiating cells. Nature 2004;432:396-401

11. O'Brien CA, Pollett A, Gallinger $S$, et al. A human colon cancer cell capable of initiating tumour growth in immunodeficient mice. Nature 2007;445:106-10
12. Uchida S, Yokoo S, Yanagi Y, et al. Sphere formation and expression of neural proteins by human corneal stromal cells in vitro. Invest Ophthalmol Vis Sci 2005;46:1620-5

13. Shmelkov SV, Butler JM, Hooper AT, et al. CD133 expression is not restricted to stem cells, and both CD133+ and CD133- metastatic colon cancer cells initiate tumors. J Clin Invest 2008; 118:2111-20

14. Ricci-Vitiani L, Lombardi DG, Pilozzi E, et al. Identification and expansion of human colon-cancer-initiating cells. Nature 2007;445:111-15

15. LaBarge MA, Petersen OW, Bissell MJ. Of microenvironments and mammary stem cells. Stem Cell Rev 2007;3:137-46

16. Sneddon JB, Werb Z. Location, location, location: the cancer stem cell niche. Cell Stem Cell 2007;1:607-11

17. Yen TH, Wright NA. The gastrointestinal tract stem cell niche. Stem Cell Rev 2006;2:203-12

18. Florek M, Haase M, Marzesco AM, et al. Prominin-1/CD133, a neural and hematopoietic stem cell marker, is expressed in adult human differentiated cells and certain types of kidney cancer. Cell Tissue Res 2005;319:15-26

19. Ray PN, Belfall B, Duff C, et al. Cloning of the breakpoint of an $\mathrm{X} ; 21$ translocation associated with Duchenne muscular dystrophy. Nature 1985;318:672-5

20. Matsumura K, Ohlendieck K, Ionasescu VV, et al. The role of the dystrophin-glycoprotein complex in the molecular pathogenesis of muscular dystrophies. Neuromuscul Disord 1993;3:533-5

21. Hoffman EP, Brown RH Jr, Kunkel LM. Dystrophin: the protein product of the Duchenne muscular dystrophy locus. Cell 1987;51:919-28

22. Ribatti D. The involvement of endothelial progenitor cells in tumor angiogenesis. J Cell Mol Med 2004;8:294-300

23. Gavina M, Belicchi M, Rossi B, et al. VCAM-1 expression on dystrophic muscle vessels has a critical role in the recruitment of human blood-derived CD133+ stem cells after intra-arterial transplantation. Blood 2006;108:2857-66
24. Yamamoto K, Kondo T, Suzuki S, et al. Molecular evaluation of endothelial progenitor cells in patients with ischemic limbs: therapeutic effect by stem cell transplantation. Arterioscler Thromb Vasc Biol 2004;24:e192-6

25. Limsuwan A, Pienvichit $P$, Limpijankit $\mathrm{T}$, et al. Transcoronary bone marrow-derived progenitor cells in a child with myocardial infarction: first pediatric experience. Clin Cardiol 2010;33:E7-12

26. Torrente Y, Belicchi M, Marchesi C, et al. Autologous transplantation of muscle-derived CD133+ stem cells in Duchenne muscle patients. Cell Transplant 2010;16:563-77

27. Bhatia R, Munthe HA, Williams AD, et al. Chronic myelogenous leukemia primitive hematopoietic progenitors demonstrate increased sensitivity to growth factor-induced proliferation and maturation. Exp Hematol 2000;28:1401-12

28. Lang P, Bader P, Schumm M, et al. Transplantation of a combination of CD133+ and CD34+ selected progenitor cells from alternative donors. Br J Haematol 2004;124:72-9

29. Stamm C, Westphal B, Kleine HD, et al. Autologous bone-marrow stem-cell transplantation for myocardial regeneration. Lancet 2003;361:45-6

30. Benchaouir R, Meregalli M, Farini A, et al. Restoration of human dystrophin following transplantation of exon-skipping-engineered DMD patient stem cells into dystrophic mice. Cell Stem Cell 2007;1:646-57

31. Flores-Ramirez R, Uribe-Longoria A, Rangel-Fuentes $\mathrm{MM}$, et al. Intracoronary infusion of CD133+ endothelial progenitor cells improves heart function and quality of life in patients with chronic post-infarct heart insufficiency. Cardiovasc Revasc Med;2010:11:72-8

32. Navarro-Sobrino M, Rosell A, Hernandez-Guillamon M, et al. Mobilization, endothelial differentiation and functional capacity of endothelial progenitor cells after ischemic stroke. Microvasc Res 2010 [Epub ahead of print]

33. Smith S, Neaves W, Teitelbaum S. Adult stem cell treatments for diseases? Science 2006;313:439 


\section{$\mathrm{CD}_{133^{+}}$cells isolated from various sources and their role in future clinical perspectives}

34. Sampaolesi M, Blot S, D’Antona G, et al. Mesoangioblast stem cells ameliorate muscle function in dystrophic dogs. Nature 2006;444:574-9

35. Mavilio F, Pellegrini G, Ferrari S, et al. Correction of junctional epidermolysis bullosa by transplantation of genetically modified epidermal stem cells. Nat Med 2006; 12:1397-402

36. Graf T, Stadtfeld M. Heterogeneity of embryonic and adult stem cells. Cell Stem Cell 2008;3:480-3

37. Negroni E, Riederer I, Chaouch S, et al. In vivo myogenic potential of human CD133+ muscle-derived stem cells a quantitative study. Mol Ther 2009; $17: 1771-8$
38. Cossu G, Bianco P.

Mesoangioblasts - vascular

progenitors for extravascular mesodermal tissues. Curr Opin Genet Dev 2003; 13:537-42

39. Grounds MD, McGeachie JK. A model of myogenesis in vivo, derived from detailed autoradiographic studies of regenerating skeletal muscle, challenges the concept of quantal mitosis. Cell Tissue Res 1987;250:563-9

40. Price FD, Kuroda K, Rudnicki MA. Stem cell based therapies to treat muscular dystrophy. Biochim Biophys Acta 2007;1772:272-83
41. Peault B, Rudnicki M, Torrente Y, et al. Stem and progenitor cells in skeletal muscle development, maintenance, and therapy. Mol Ther 2007;15:867-77

\section{Affiliation}

Mirella Meregalli, Andrea Farini, Marzia Belicchi \& Yvan Torrente

${ }^{\dagger}$ Author for correspondence Stem Cell Laboratory,

Department of Neurological Sciences, Università degli Studi di Milano,

Fondazione IRCCS Cà Granda Ospedale Maggiore Policlinico,

Centro Dino Ferrari, Italy Tel: +39 02 55033874; Fax: +3902 50320430;

E-mail: yvan.torrente@unimi.it 